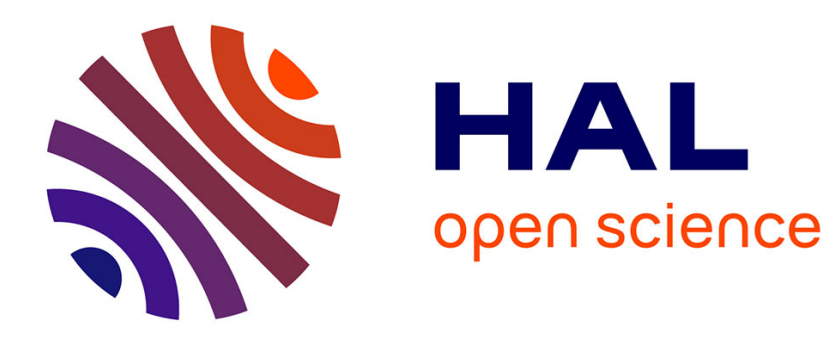

\title{
Bayesian Inference for Modal Identification in Ducts with a Shear Flow
}

Rémi Roncen, Estelle Piot, Fabien Mery

\section{To cite this version:}

Rémi Roncen, Estelle Piot, Fabien Mery. Bayesian Inference for Modal Identification in Ducts with a Shear Flow. 2019. hal-02139738

\section{HAL Id: hal-02139738 \\ https://hal.science/hal-02139738}

Preprint submitted on 25 May 2019

HAL is a multi-disciplinary open access archive for the deposit and dissemination of scientific research documents, whether they are published or not. The documents may come from teaching and research institutions in France or abroad, or from public or private research centers.
L'archive ouverte pluridisciplinaire HAL, est destinée au dépôt et à la diffusion de documents scientifiques de niveau recherche, publiés ou non, émanant des établissements d'enseignement et de recherche français ou étrangers, des laboratoires publics ou privés. 


\title{
Bayesian Inference for Modal Identification in Ducts with a Shear Flow
}

\author{
R. Roncen, E. Piot, and F. Mery \\ ONERA /Département Multi-Physique pour l'Énergétique, Université de Toulouse, F-31055, Toulouse, France
}

\begin{abstract}
An in-duct modal decomposition technique is described. The basis for the technique is to consider the decomposition as an inference problem. Using transfer function measurements at the duct walls, a Bayesian inference is conducted to evaluate the acoustic modal coefficients in the presence of uncertainties. These uncertainties encompass model errors, microphone measurements error, and uncertainty on the flow profile. The formalism of the direct problem of modal decomposition in a ducted shear flow is first developed. The case of a circular cross-section duct is then treated without and with a flow, using synthetic noisy signals for the inference problem.
\end{abstract}

\section{Introduction}

Prediction and reduction of noise are ubiquitous goals in many engineering fields such as aeronautics, hydrodynamics and transport, to either increase operational capacity and/or minimize the impact on the environment. The testing of the different design elements pertaining to these product candidates (acoustic liners, scale models, etc.) usually rely on measurements performed in ducts carrying energy in the form of a flow and/or a sound field [1, 2, 3, 4, 5, 6, In this context, it is necessary to characterize the structure of the noise that propagates within the duct in the presence of a shear flow.

A modal decomposition technique allows such a characterization of the acoustic field structure (modes) inside a duct. A wide range of decomposition methods are already available, ranging from source localization techniques such as beamforming [7, 8, singular values decomposition [9, deconvolution methods [10] and pseudo-inverse resolution of a linear problem. The foundation of the latter procedure was proposed by Åbom [11, who first recognized that the two-microphone transfer function method by Chung and Blaser [12] could be extended to non plane wave modes.

In Åbom's method, the duct eigenfunctions and eigenvalues are assumed known exactly. For $N$ propagating modes in the duct, the pressure field needs to be observed at $M \geq 2 N$ independent measurement positions to separate the incident and reflected parts of the pressure field. A pseudo-inverse of the modal matrix $\left(L_{2}\right.$ minimization) then allows the retrieval of all the modal coefficients. Little account was given regarding the resulting uncertainties in the modal decomposition technique [13, particularly in the presence of a shear flow or when a high number of modes propagate in the duct. Abom quantified the influence of a $1 \%$ error on the input measurements on the identification, showing a resulting error in modal amplitude of about the same order of magnitude. It was argued that a uniform flow would have no influence on the technique validity, but that non-uniformity effects could introduce errors in the modal decomposition. While these errors were shown to be negligible for a modal decomposition based on measured radial profiles [14, most practical solutions include wall-mounted microphones, where the effect of mode shape distortion might be relevant.

The aim of this work is to amend Åbom's procedure by taking into account uncertainties directly during the modal decomposition. To that end, the decomposition procedure is recast as an inference problem, where Bayes' theorem is used to update the information on the modal structure, given a new observation. This observation consists, as previously, as wall-mounted microphone measurements. Compared to Åbom's procedure, the case of

*Corresponding author: remi.roncen@onera.fr 
the presence of a shear flow inside the duct is considered, with an uncertain flow profile. This results in the duct modes wavenumbers not being exactly known.

The present work is organized as follows. In Section 2, the in-duct modal representation of acoustic fields is detailed, along with the numerical technique that allows the retrieval of the duct wavenumbers in the presence of a shear flow. In Section 3, the statistical Bayesian modal identification technique is developed. Identification results are given in Section 4 giving rise to new questions that are subsequently answered. A conclusion is then drawn in Section 5 .

\section{Modal acoustics in ducts with flow}

In this section, the general representation of in-duct pressure is recalled. The computing procedure of the duct wavenumbers is then given, accounting for the presence of a shear grazing flow.

\subsection{Modal representation}

A circular cylindrical duct of radius $R_{\text {duct }}$ is considered. $x$ is the axial coordinate, which increases from left to right. $(r, \theta)$ are the radial and azimuthal coordinates, respectively. Assuming a finite number of cut-on modes, an ideal fluid, a small amplitude variation (linear acoustic theory valid), then the pressure field inside the duct can be expressed as a Fourier-Bessel expansion [15] ( $e^{+j \omega t}$ convention)

$$
p(x, r, \theta)=\sum_{m=-\infty}^{\infty} \sum_{n=0}^{\infty}\left[A_{m, n} e^{-\mathrm{j} k_{m, n}^{+} x}+B_{m, n} e^{+\mathrm{j} k_{m, n}^{-} x}\right] \Psi_{m, n}(r) e^{\mathrm{j} m \theta}
$$

with the azimuthal mode $m \in \mathbb{Z}$, and $n \in \mathbb{N}_{0}$ the radial mode order. $k_{m, n}^{ \pm}$are the axial wavenumbers ( + for right going incident wave, - for left going reflected wave); $A_{m, n}, B_{m, n}$ are the associated modal amplitudes. By convention in this work, the plane wave mode is $(m, n)=(0,0)$, and not $(0,1)$ as can be found sometimes [16]. In this work, the modes are normalized by arbitrarily setting $\Psi_{m, n}\left(r=R_{\text {duct }}\right)=1$, since all measurements are performed at the wall.

\subsection{Governing equations}

An inviscid perfect gas is considered inside the duct. The Euler equations read

$$
\begin{aligned}
\frac{\partial \rho}{\partial t}+\nabla \cdot(\rho \boldsymbol{v}) & =0, \\
\rho \frac{\mathrm{D} \boldsymbol{v}}{\mathrm{D} t}+\boldsymbol{\nabla} p & =0, \\
\frac{\mathrm{D} s}{\mathrm{D} t} & =0,
\end{aligned}
$$

where $p$ is the pressure, $\rho$ the density, $s$ the entropy; $\boldsymbol{v}$ is the velocity with axial, radial and azimuthal components $u, v$ and $w$, respectively. The convective derivation is written $\mathrm{D} / \mathrm{D} t=\partial / \partial t+(\boldsymbol{v} \cdot \boldsymbol{\nabla})$. The flow field is decomposed into a static field (subscript 0) and a small-amplitude unsteady perturbation (tilde symbol):

$$
(\boldsymbol{v}, p, \rho)=\left(\boldsymbol{v}_{0}, p_{0} / \gamma, \rho_{0}\right)+(\tilde{\boldsymbol{v}}, \tilde{p}, \tilde{\rho})
$$

with $\gamma=c_{p} / c_{v}$ the gas adiabatic index. 
As a simplifying assumption, the flow is considered to be parallel to the propagation axis:

$$
\begin{aligned}
& u_{0}=u_{0}(r), \\
& v_{0}=0 \\
& w_{0}=0 \\
& p_{0}, \rho_{0} \text { constant. }
\end{aligned}
$$

Dimensionless quantities are hereafter defined as: $x \rightarrow x / R_{\text {duct }}, r \rightarrow r / R_{\text {duct }}, t \rightarrow t / t_{0}, \boldsymbol{v} \rightarrow \boldsymbol{v} / c_{0}, \rho \rightarrow \rho / \rho_{0}$, $p \rightarrow p / p_{\text {ref }}$, with $c_{0}=\sqrt{\gamma p_{0} / \rho_{0}}, t_{0}=R_{\text {duct }} / c_{0}, p_{\text {ref }}=\rho_{0} c_{0}^{2}$.

The linearized form of the Euler equations (LEE) are obtained using the small-amplitude perturbations. If a harmonic excitation is considered, the field decomposition under the harmonic form can be sought for any field quantity as

$$
\tilde{\varphi}(r, x, t)=\tilde{\varphi}(r) e^{\mathrm{j} \omega t \pm \mathrm{j} k_{m, n}^{ \pm} x-\mathrm{j} m \theta},
$$

where $\omega=2 \pi f R_{\text {duct }} / c_{0}$ is the dimensionless angular frequency (with $f$ the frequency in $\mathrm{Hz}$ ). Using the entropy equation and the homentropicity condition (adiabatic reversible flow and uniform entropy at infinite upstream of the flow), one obtains $p=\rho$ and the entropy term is hereafter dropped from the equations. The dimensionless quantities $(\tilde{u}, \tilde{v}, \tilde{w}, \tilde{p})$ are unknown functions of $r$. The LEE can then be written

$$
\mathcal{L}(k, \omega) \cdot \mathcal{X}=0
$$

where $\mathcal{L}$ is a linear operator defined as a function of a wavenumber $k$, and $\omega$, for a given azimuthal wavenumber $m$, as

$$
\mathcal{L}(k, \omega)=\left(\begin{array}{cccc}
\mathrm{j}\left(\omega-k M_{0}\right) & -\mathrm{j} k & \mathrm{~d} / \mathrm{d} r+1 / r & -\mathrm{j} m / r \\
-\mathrm{j} k & \mathrm{j}\left(\omega-k M_{0}\right) & M_{0}^{\prime} & 0 \\
\mathrm{~d} / \mathrm{d} r & 0 & \mathrm{j}\left(\omega-k M_{0}\right) & 0 \\
-\mathrm{j} m / r & 0 & 0 & \mathrm{j}\left(\omega-k M_{0}\right)
\end{array}\right),
$$

applied to the vector $\mathcal{X}=(\tilde{u}, \tilde{v}, \tilde{w}, \tilde{p})$, and where $M_{0}$ stands for the dimensionless axial velocity, i.e. the axial Mach number, and is a function of $r$. A general boundary condition representing either a rigid wall or an acoustic treatment is given via the definition of a material surface acoustic impedance $\zeta(\omega)$, here a dimensionless quantity $\left(\times \rho_{0} c_{0}^{2}\right.$ to re-obtain a dimensional quantity). If the mean velocity field cancels at the wall, the classical expression between unsteady harmonic pressure and velocity fields is, in the frequency domain,

$$
\tilde{p}(1)=\zeta \tilde{v}(1),
$$

where 1 stands for $R_{\text {duct }} / R_{\text {duct }}$. A value of $\zeta \rightarrow \infty$ represents a rigid wall configuration, and is the case retained in this work since modal decomposition is usually performed in rigid wall ducts. Numerically, we enforce $\tilde{v}=0$ at the wall to avoid Inf values in the code.

Since no obvious solution exists in the case of a non-uniform flow for Eq. 6, a numerical solution can be sought, provided that the problem be discretized.

\section{$2.3 \quad$ Numerical implementation}

The eigenvalue problem (6)-(8) is solved with a numerical strategy similar to the one used in Ref. [17]. A Chebychev polynomial basis $\left(T_{k}\right)_{k \in\left[0, N_{\text {Cheb }}\right]}$ of order $N_{\text {Cheb }}$ is used to decompose the LEE problem (6)-(8). The physical reduced variable $r \in[0,1]$ and the Chebychev variable $\kappa \in[-1,1]$ are related by

$$
r(\varsigma)=\frac{\lambda(1-\kappa)}{1+2 \lambda+\kappa}
$$


with $\lambda$ a fixed parameter, controlling the collocation point clustering. These are defined by

$$
\kappa_{i}=\cos \left(\frac{\pi i}{N_{\text {Cheb }}}\right), \quad i \in\left[0, N_{\text {Cheb }}\right]
$$

which naturally clusters the points near the boundaries -1 and 1 . Setting

$$
\lambda=-(1+\epsilon)
$$

with $0<\epsilon \ll 1$ increases the resolution near the wall, as shown in Fig 1. Hereafter, the values are fixed at $N_{\text {Cheb }}=50$ and $\lambda=-1.05$ to achieve mesh independence for the flows considered in this work.

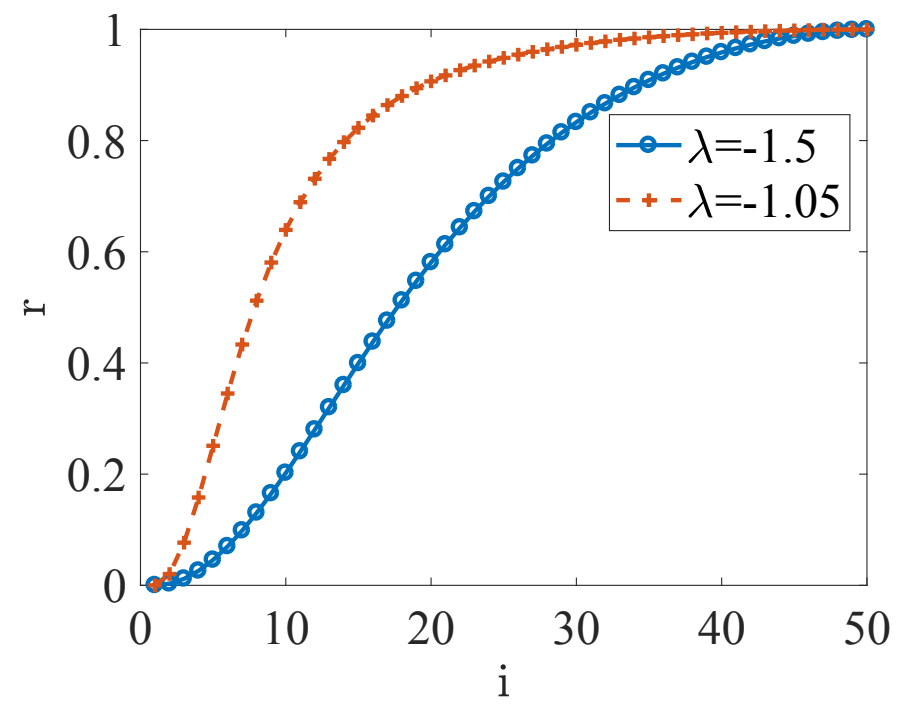

Figure 1: Mesh clustering for different $\lambda$, with $N_{\text {Cheb }}=50$

A derivation matrix $\boldsymbol{A}$ exists to derive a function $F$ (that must be a linear combination of Chebychev polynomials) with respect to $\varsigma$ on the collocation points $\varsigma_{i}$ :

$$
\frac{\mathrm{d} F}{\mathrm{~d} \varsigma}\left(\varsigma_{i}\right)=\sum_{j=0}^{N_{\text {Cheb }}} \boldsymbol{A}_{i j} F\left(\varsigma_{j}\right) .
$$

This can be used to represent the $\mathrm{d} / \mathrm{d} r$ terms in Eq. 7. after a suitable change of variable. The solution vector $\mathcal{X}=(\tilde{u}, \tilde{v}, \tilde{w}, \tilde{p})$ is decomposed onto the Chebychev basis, resulting in a discrete representation of the solution array

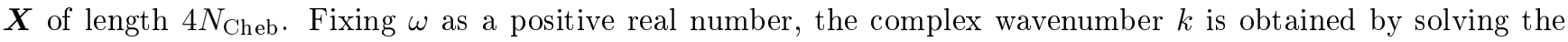
reformulated eigenvalue problem

$$
[\boldsymbol{D}+k \boldsymbol{Q}] \cdot \boldsymbol{X}=0
$$

where $\boldsymbol{D}$ and $\boldsymbol{Q}$ are $4 N_{\text {Cheb }} \times 4 N_{\text {Cheb }}$ matrices, extracted from the discretized version of the direct operator $\mathcal{L}$ of Eq. 7 and associated boundary conditions. To avoid reassembling the $\boldsymbol{D}$ matrix at each different value of $\omega$, we separate $\boldsymbol{D}$ as $\boldsymbol{D}=\boldsymbol{D}_{0}+\omega \boldsymbol{D}_{1}$, with $\boldsymbol{D}_{0}$ and $\boldsymbol{D}_{1}$ elementary matrices that only need being computed once.

The solutions $(k, \boldsymbol{X})$ of Eq. 13 are the modes axial wavenumber $k_{m, n}$ and radial eigenfunction $\boldsymbol{X}_{m, n}$, respectively. From the eigenfunctions $\boldsymbol{X}_{m, n}$, one can obtain the pressure eigenfunction $\Psi_{m, n}\left(r_{i}\right)$ by extracting every fourth component (since $X=\left(\tilde{u}_{i=0}, \tilde{v}_{i=0}, \tilde{w}_{i=0}, \tilde{p}_{i=0}, \ldots \tilde{u}_{i=N_{\text {Cheb }}}, \tilde{v}_{i=N_{\text {Cheb }}}, \tilde{w}_{i=N_{\text {Cheb }}}, \tilde{p}_{i=N_{\text {Cheb }}}\right)$ ).

\subsection{Influence of a shear flow}

In most engineering applications, a shear flow is present in the duct that needs modal characterization. However, the flow profile is only known to some extent, resulting in only approximate values of the wavenumbers calculated 
using the numerical implementation of Sec. 2.3. In this work, the hyperbolic velocity profile defined in Rienstra and Vilensky [18] and used in Khamis and Brambley [19] is considered, which depends on two parameters: the mean flow Mach number $M_{b}$ and a shear parameter $\delta \in(0,1]$, as

$$
\begin{aligned}
& M_{0}(r)=\tanh \left(\frac{1-|r|}{\delta}\right)+\left(1-\tanh \left(\frac{1}{\delta}\right)\right) \cdot\left(\frac{|r|}{\delta}\left(1+\tanh \left(\frac{1}{\delta}\right)\right)+1+|r|\right) \cdot(1-|r|), \\
& M_{0}(r) \leftarrow \frac{M(r)}{\int_{0}^{1} M(r) \mathrm{d} r} M_{b}
\end{aligned}
$$

where the second step is a normalization fixing the average (bulk) value $M_{b}$ in the case of incompressible flow. Taking a fixed $M_{b}=0.2$, Fig. 2 shows the evolution of the flow profile as $\delta$ increases from a low value (high shear) to a high value (low shear, Poiseuille-like profile). Note that $\delta$ can take values higher than one, but that in practice the profile remains quite similar to the case $\delta=1$. For a high shear, the mesh used in Sec. 2.3 must be refined close to the wall boundary, i.e., $\epsilon$ must be chosen small in the expression of $\lambda$ in Eq. 11. The wavenumbers associated to different modes are shown in Fig. 3 for different values of $\delta$; only the modes related to the right going waves $k_{m, n}^{+}$are shown; $k_{r}$ (resp. $k_{i}$ ) stands for the real (resp. imaginary) part of the wavenumber. Increasing $\delta$ (reducing the shear) translates the modes wavenumbers to the right of the graph, except for the first radial mode $(m, n)=(0,1)$, which remains almost the same whatever the shear. For left going waves, the conclusions on $k_{m, n}^{-}$are similar: decreasing the shear shifts the negative real parts of $k_{m, n}^{-}$to the right as well.

Since the flow profile plays a role in determining the $k_{m, n}$, uncertainties on the shape of the flow profile have an influence on the determination of the modal content. In this work, the flow profile is therefore taken as unknown, and the additional parameters $M_{b}$ and $\delta$ are part of the identification process.

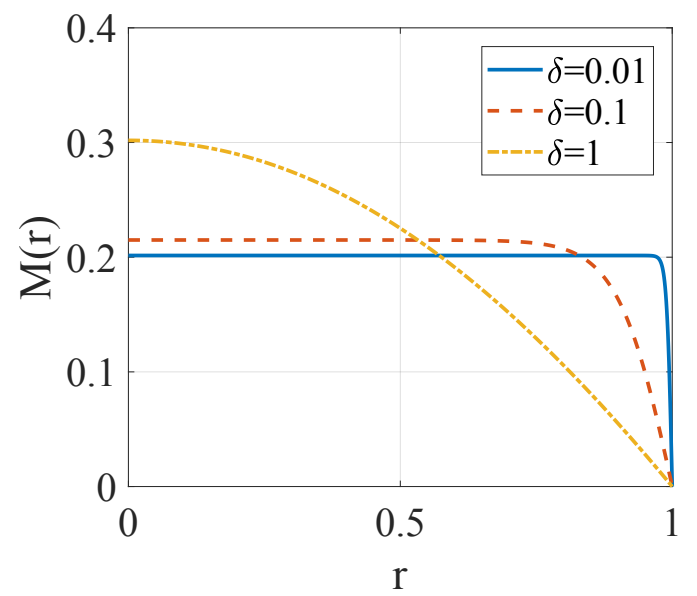

Figure 2: Evolution of the flow profile as a function of $\delta$, for $M_{b}=0.2$

\section{Modal identification}

In this section, we first recall the modal decomposition technique of $\AA$ bom. The method is then recast as an inference problem, where Bayes theorem is used to compute the probability density functions of the modal amplitudes, which are the quantities of interest.

\subsection{Deterministic Inverse problem}

A generalization of the two-microphone method [20] was proposed by Åbom [11] to sort the right running and left running waves, for each mode (i.e., finding the $A_{m, n}$ and $B_{m, n}$ of Eq. 1). If one wishes to identify $N$ propagating modes, then one requires to find $2 \times N$ unknowns $\left(A_{m, n}\right.$ and $\left.B_{m, n}\right)$. It thus comes to reason that at least $M \geq 2 N$ independent equations need to be available when trying such an identification. These equations usually come in the 


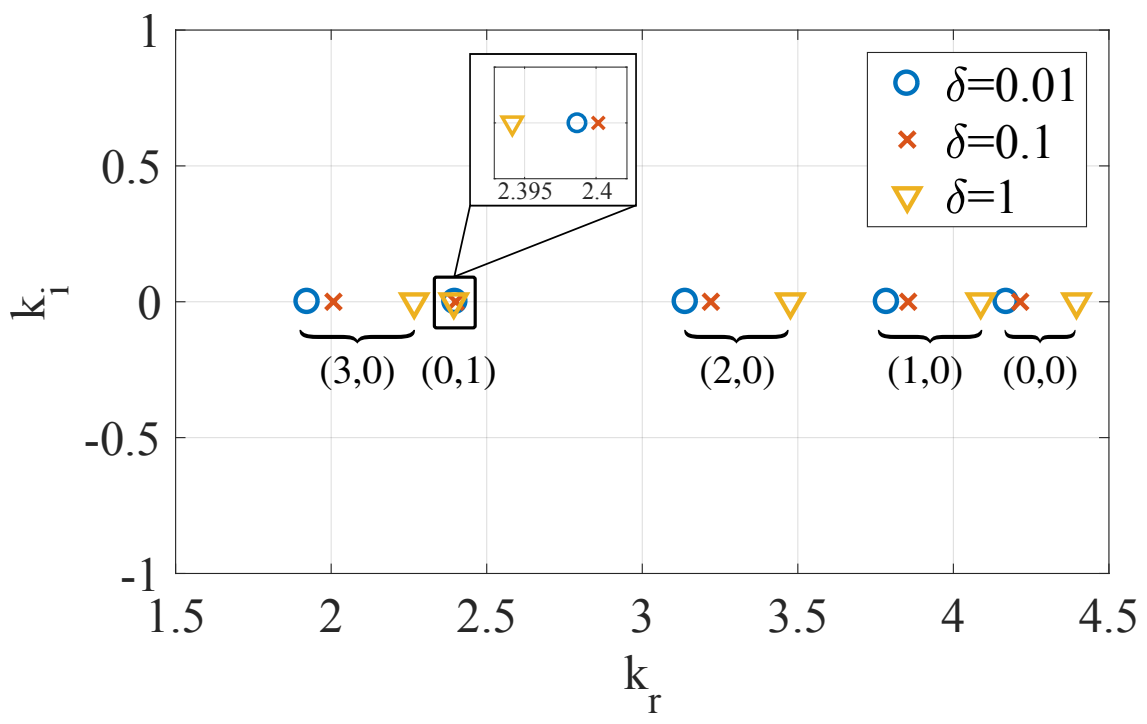

Figure 3: Evolution of the wave numbers as a function of $\delta$, for $M_{b}=0.2$ at $\omega=5$

form of pressure measurements at the wall, but "in duct" measurements could be used as well if available. Using a matrix formulation, the result of such a measurement, at a given frequency, is written as

$$
\hat{\boldsymbol{p}}=\boldsymbol{M} \hat{\boldsymbol{a}}
$$

where $\hat{\boldsymbol{p}}$ is a $[M \times 1]$ column vector containing the acoustic pressure measurements, $\boldsymbol{M}$ is a $[M \times 2 N]$ modal matrix containing modal terms such as $\left[e^{-j k_{m, n}^{+} x_{\text {mic. }}}, e^{+j k_{m, n}^{-} x_{\text {mic. }}}\right] \Psi_{m, n}\left(r_{\text {meas }}\right) e^{j m \theta_{\text {mic. }}}$, and $\hat{\boldsymbol{a}}$ is the $[2 N \times 1]$ column vector of unknown modal amplitudes. From Eq. 15 one then gets $\hat{\boldsymbol{a}}$ as

$$
\hat{\boldsymbol{a}}=M^{-1} \hat{\boldsymbol{p}}
$$

where the inverse is understood as a Moore-Penrose pseudoinverse when $M>2 N$. That is, $\hat{\boldsymbol{a}}$ can be seen as the solution of an optimization problem, where the least-mean square minimum is sought:

$$
\hat{\boldsymbol{a}}=\arg \min _{\hat{\boldsymbol{b}}}\left(\|\boldsymbol{M} \hat{\boldsymbol{b}}-\hat{\boldsymbol{p}}\|_{2}\right) \text {. }
$$

The main issue with this approach is the impossibility to account for uncertainties in the measured pressure $\hat{\boldsymbol{p}}$, or other variables (flow profile, model errors, etc). One way to circumvent the issue is to recast the identification procedure as a statistical inference problem. The identified modes are then considered as unknown random variables, whose probability density functions translate our state of knowledge on the parameters. This is the topic of the next section.

\subsection{Bayesian inference for modal uncertainty quantification}

\subsubsection{Bayes theorem applied to modal identification}

In the context of inverse problems for parameter estimation, statistical inference in the Bayesian framework consists of recasting parameters of interest as random variables associated with probability densities encompassing the information one has on the parameters [21, Chap. 8]. A new experimental data $p^{\text {obs }}$ is observed (here pressure measurements at the wall), and this information is used to update our state of knowledge. $p^{\text {obs }}$ is considered as the realization of a multi-variate random variable $P^{\text {obs }}$. The degree of knowledge about the true value of the unknown parameters $\hat{A}=\left(A_{m, n}, B_{m, n}\right)$, with realizations $\hat{a}$, is represented by the marginal posterior density $\pi\left(\hat{a} \mid p^{\text {obs }}\right)$, the 
new quantity of interest. Using Bayes theorem, one has

$$
\pi\left(\hat{a} \mid p^{\mathrm{obs}}\right)=\frac{\pi\left(p^{\mathrm{obs}} \mid \hat{a}\right) \pi_{0}(\hat{a})}{\pi\left(p^{\mathrm{obs}}\right)}
$$

where $L(\hat{a}) \equiv \pi\left(p^{\text {obs }} \mid \hat{a}\right)$ is the likelihood function, representing the probability that the experience be observed, given a particular set of model parameters; $\pi_{0}(\hat{a})$ is the prior probability, representing all the information obtained on $\hat{a}$ prior to the new observation (i.e., previous knowledge on the modal content of an experiment). Finally, $\pi\left(p^{\text {obs }}\right)$ is a scaling constant not calculated in practice. Details on the likelihood and prior modeling are given hereafter.

\subsubsection{Likelihood}

The likelihood function represents the mechanism through which information provided by the observation is incorporated into the posterior density. The physical correlations between the model parameters $q$ and the experimental data, as predicted by a physical model (here the LEE), are represented by a deterministic forward operator $f$ defined as

$$
f_{i}(\hat{a})=\hat{p}\left(x_{i}, r_{i}, \theta_{i}\right), \quad i=1, \ldots, M
$$

where $\hat{p}_{t}\left(x_{i}\right)$ is a numerical pressure signal at position $\left(x_{i}, r_{i}, \theta_{i}\right)$, and $M$ is the number of measurements. Due to both the observation and the modeling being corrupted by uncertainties, it is legitimate to consider that the measurement $P^{\text {obs }}$ be related to the deterministic operator $f$ by a relationship of the type

$$
P_{i}^{\mathrm{obs}}=f_{i}(\hat{a})+\varsigma_{i}, \quad i=1, \ldots, n
$$

where $\varsigma_{i}$ is a random variable representing measurement errors at the $i^{\text {th }}$ measurement. In the case where the measurement errors are independent and identically distributed and $\varsigma_{i} \sim \mathcal{N}\left(0, \sigma_{i}^{2}\right)$, then

$$
P_{i}^{\text {obs }} \sim \mathcal{N}\left(f_{i}(\hat{a}), \sigma_{i}^{2}\right)
$$

which leads to

$$
\pi\left(p_{i}^{\mathrm{obs}} \mid \hat{a}\right)=\frac{1}{\sqrt{2 \pi \sigma_{i}^{2}}} e^{-\left[p_{i}^{\mathrm{obs}}-f_{i}(\hat{a})\right]^{2} / 2 \sigma_{i}^{2}} .
$$

When considering all the measurement points (at different time steps) as independent uncorrelated measurements, the likelihood becomes

$$
\pi\left(p^{\mathrm{obs}} \mid \hat{a}\right)=\prod_{i=1}^{n} \pi\left(p_{i}^{\mathrm{obs}} \mid \hat{a}\right) .
$$

where $\sigma_{i}^{2}$ denotes the noise variance of the $i^{\text {th }}$ microphone measurement. The larger the value of $\sigma_{i}^{2}$, the larger the estimated uncertainty on the observation. In this work, it is assumed unknown and is a parameter to identify (included in $\hat{a}$ ), but remains the same for all measurement points. For numerical reasons, the logarithm of the likelihood is used instead of Eq. 23. As is the case for classical inverse problems where a cost function has to be minimized in order to retrieve the parameters of interest, the likelihood can be viewed as the probabilistic equivalent of such a quantity, measuring the probability of the experience being observed, for a given set of parameters.

\subsubsection{Prior model}

The prior probability $\pi_{0}(\hat{a})$ incorporates the information obtained on the model parameters independently of the observation $p^{\text {obs }}$ used during the inference. It reflects the user's prior insight into the problem, generalizing the classical constraints used during deterministic inversion, assigning weights to the most probable values. When no prior information is available or if prior modeling is of questionable accuracy, a wide-range, non-informative improper homogeneous density on the parameters supports represents well one's ignorance and merely sets boundaries for each parameter, as in classical deterministic inverse problems. In the present work, prior independence is first 
assumed among the unknown parameters, setting

$$
\pi_{0}(\hat{a})=\chi_{\left[\hat{a}_{\min }, \hat{a}_{\max }\right]}(\hat{a}),
$$

where $\chi$ is a uniform density between $\hat{a}_{\min }$ and $\hat{a}_{\max }$, and 0 outside these bounds.

\subsubsection{Gaussian hypothesis}

Further assuming that the unknown variables have a Gaussian density, it is sufficient, when solving Eq. 18 , to find the maximum of $\pi\left(p^{\mathrm{obs}} \mid \hat{a}\right) \pi_{0}(\hat{a})$ (since the denominator is constant). This quantity is coined Maximum A Posteriori (MAP) in the literature [21, Chap. 8] . With a Gaussian hypothesis and non-informative priors, most Bayesian estimators (Maximum A Posteriori, Maximum Likelihood Estimator and mean) are equivalent. One only has to solve an optimization problem where the left hand side of Eq. 18 is maximized. The point of maximum yields the MAP of the unknown variables. Then, second order moments (variance) can be quickly calculated via the Hessian matrix, evaluated at the point of maximum. Let $\hat{a}_{\text {optim }}$ be the point of maximum, and $\mathcal{H}$ the Hessian of $\pi\left(p^{\mathrm{obs}} \mid \hat{a}\right) \pi_{0}(\hat{a})$ evaluated at $\hat{a}_{\mathrm{optim}}$. Then the covariance matrix is $S=\mathcal{H}^{-1}$ and the standard deviation of the parameters are the square root of the diagonal elements of $S$.

Note that the Gaussian hypothesis is quite strong. Before using it safely, Markov-Chain Monte-Carlo simulations were performed (using the MT_DREAM_ZS algorithm of Laloy and Vrugt [22]) in order to explore the posterior densities of the parameters, and it was verified that the hypothesis is indeed adequate. In the remainder of this work, the MAP estimate is obtained by optimizing the numerator of Eq. 18 and the covariance matrix is calculated as explained above.

\section{Identification results}

Different identification results obtained using synthetically generated signals are presented in this section, to validate the method and give some hindsight about modal detection antenna design.

\subsection{Case without flow}

In the case when there is no shear flow within the duct $\left(M_{b}=0\right)$, the inference strategy is straightforward. A synthetic signal is first generated at 100 fictive microphone locations, and a zero mean Gaussian noise is then added to pollute the signals. The microphones are spread at random locations, covering a duct length equal to $3 R_{\text {duct }}$. This high number of microphones is representative of the CANNELLE bench used jointly by Airbus and ONERA [23] to identify higher order modes in nacelle configurations. A noise of standard deviation 0.05 is added to both the real and imaginary parts of the numerically generated pressure signals, which results in $\sigma=0.05 \sqrt{2}$. At each frequency, the right going incident wave is taken as $A_{m, n}=(1+\mathrm{j}) / \sqrt{2}$ so as to have an amplitude $\left|A_{m, n}\right|=1$ and a phase $\angle_{m, n}^{+}=\pi / 4$, while the left going wave is taken as $B_{m, n}=(1+0.2 \mathrm{j}) / 2 \sqrt{2}$ so has to have $\left|B_{m, n}\right| \approx 0.36$ and $\angle_{m, n}^{-} \approx \pi / 16$.

Identification results are shown in Fig. 4 . At each frequency $\omega$, all the modal coefficients $\left(A_{m, n}, B_{m, n}\right)$ are inferred, if the associated mode is cut-on. However, for conciseness, only the amplitudes of the right going waves having a positive azimuthal order $m$ are shown (conclusions remain the same for negative azimuthal orders, for left going waves, and for the phase). In addition, the experimental standard deviation $\sigma$ intervening in Eq. 22 is also taken as an unknown parameter to be calibrated. The prior bounds are taken as wide uniform laws, so as to not constrain the inference.

The results are displayed in the form of confidence intervals (CI) containing respectively $95 \%$ and $63 \%$ of the probabilistic weight of the posterior densities. The MAP is also shown (here, it is equivalent to the mean), alongside the true value that was used to generate the synthetic signals. Overall, all the modal coefficients are well identified, in both the amplitudes and phase, in the full frequency range studied here. The same conclusion is drawn for the standard deviation $\sigma$, which is a far-reaching result: for a given experimental measurement, the error that is 
representative of the mismatch between the model and the experimental observation can be inferred directly from the Bayesian inference strategy.
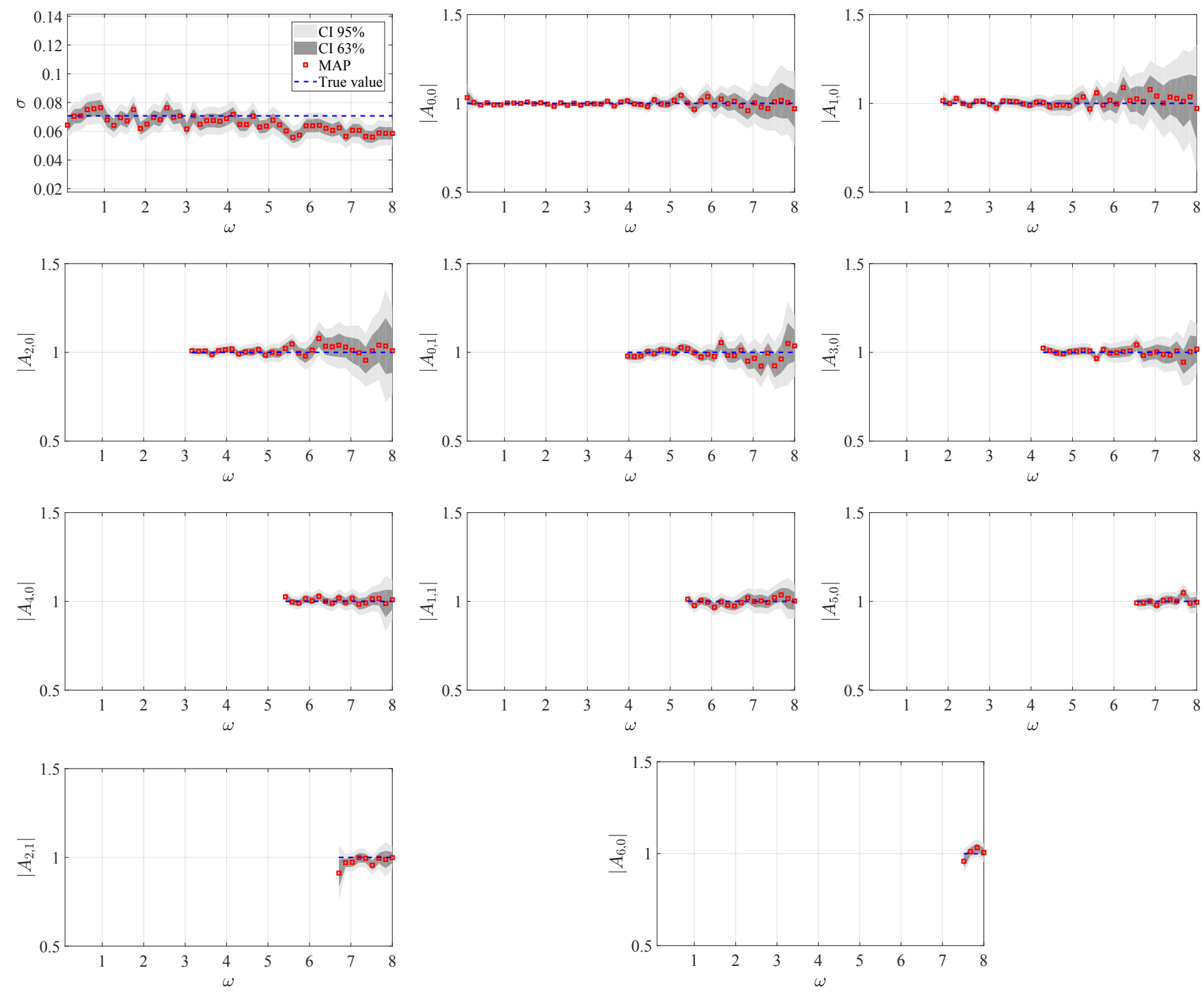

Figure 4: Inference results in the no flow case

Interestingly, the credibility intervals seem to increase as the frequency increases for low order modes, while higher order mode coefficients display a lower uncertainty at these frequencies. To investigate this phenomenon, the correlation matrix (absolute value of the Pearson coefficient) is shown in Fig. 5 at $\omega=2.36$ and $\omega=8$. At $\omega=8$, there are 19 modes considered in the inference, which results in $19 \times 4=76$ parameters $\left(\left|A_{0,0}\right|, \angle_{0,0}^{+},\left|B_{0,0}\right|, \angle_{0,0}^{-}, \ldots,\right)$, yielding a $76 \times 76$ symmetric correlation matrix. A zoom of the correlation between the parameters of the first three modes is provided in Fig. 5 a at $\omega=8$. Only the absolute correlation is displayed, with hotter colors associated to a high correlation. It appears in Fig. 5 a that the parameters associated to lower order modes are strongly correlated between each other (upper left side of the matrix), while those of higher order modes are not (lower right side). Here, the correlation is not a physical one, but is merely an information related to both the model and the observation. The influence of two correlated parameters on the model, evaluated at the measurement location, is similar. For instance, in the case of two perfectly positively correlated parameters (correlation is 1), an increase in both parameters could leave the likelihood function unchanged, making it impossible to separate these parameters during the inference [21, Chap. 8]. Here, some information can still be extracted from the experimental observation 
and the parameters can be identified, but the strong correlations make it more difficult, which translates in higher uncertainties for the lower order modes. By contrast, the correlation matrix obtained at $\omega=2.36$ in Fig. $5 \mathrm{~b}$ displays lower correlation values, meaning that at lower frequencies the correlation phenomenon is not present. This results in a less uncertain identification of the first modes (combined with the fact that there are less parameters to identify).

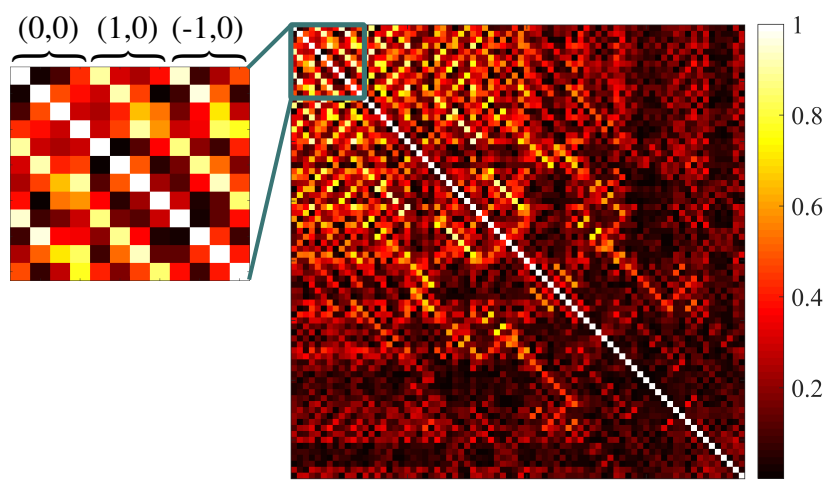

(a) $\omega=8$

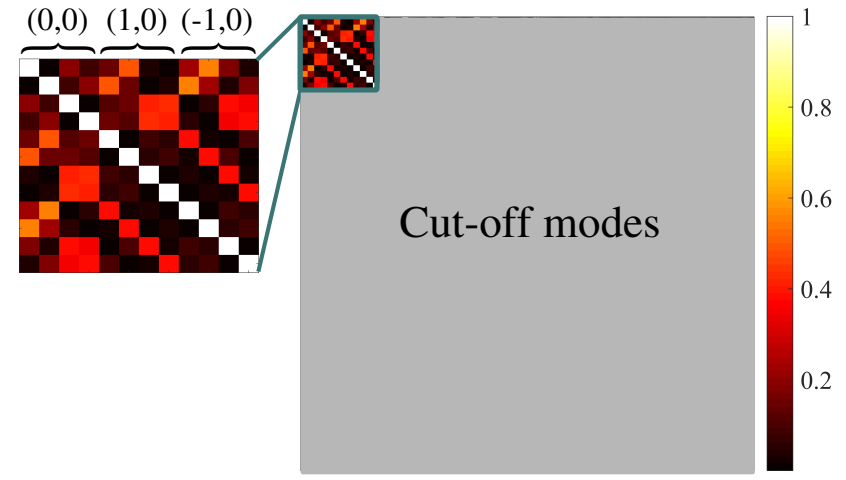

(b) $\omega=2.36$

Figure 5: Correlation matrices in the no flow case

To better explain why correlations are present at high frequencies and not at lower frequencies, the wavenumbers are plotted at different values of $\omega$ in Fig. 6a here with a radial order $n=1$ for conciseness. The wavenumbers are each rescaled by $k_{0,0}^{+}=\omega$ in order to observe the relative spacing between wavenumbers, as the frequency increases. It appears that the modes wavenumbers tend to cluster as the frequency increases, which is confirmed in Fig. 6b where the relative difference between a given mode and the previous cut-on mode

$$
\Delta_{k}=\frac{\operatorname{real}\left(k_{m, n}^{+}-k_{m-1, n}^{+}\right)}{k_{0,0}}
$$

is shown as a function of the frequency.

Therefore, the higher the frequency, the higher the clustering of the modes wavenumbers, which explains why the correlation values get higher for a given set of modes when the frequency is increased.

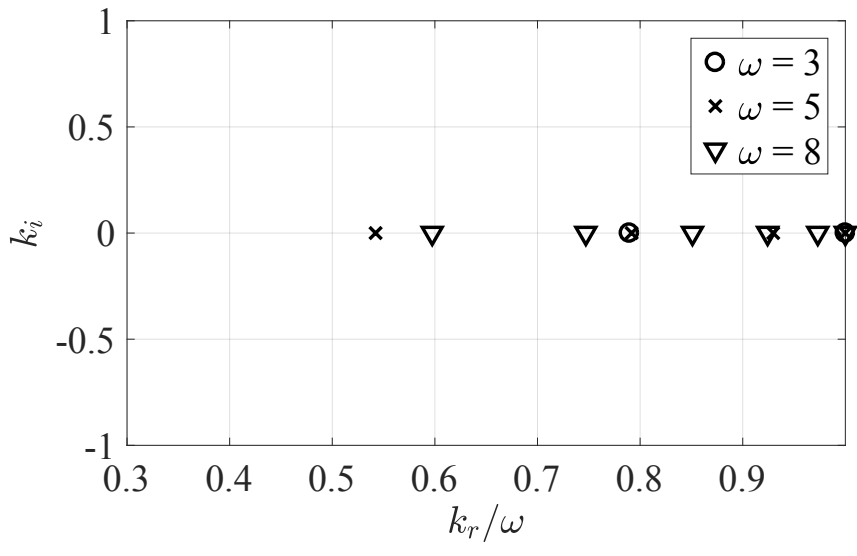

(a) Clustering of wavenumbers as the frequency increases

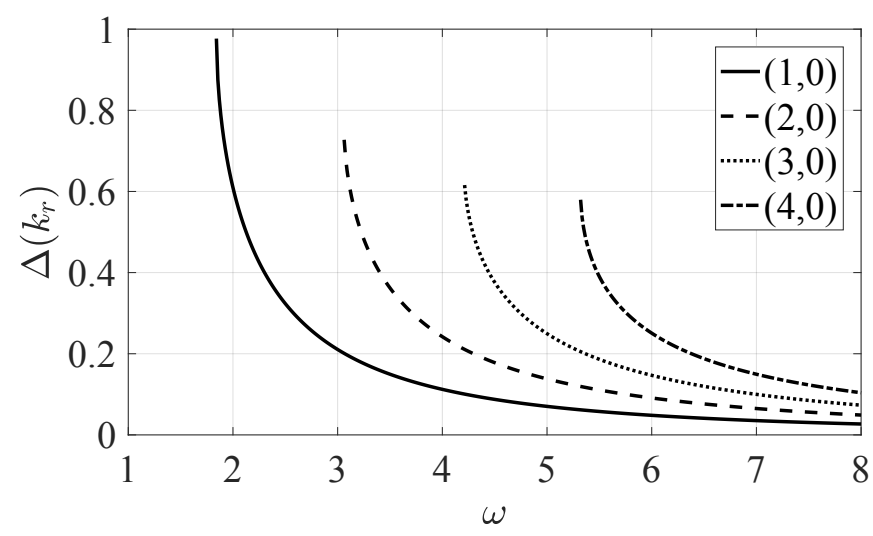

(b) Distance between modes as a function of the frequency

Figure 6: Modal wavenumber repartition in the complex plane 


\subsection{Influence of the observational sparsity and spread}

In the previous section, two parameters were fixed during the inference: the number of microphones $N$ and their spread $L$ on the longitudinal axis. These two parameters are of interest for practical purposes, where one wants to maximize the information gain at a lower cost and complexity (number of microphones). As an example and for the plane wave mode $(m, n)=(0,0)$, the uncertainty at very low $\omega$ increases (see Fig. 4). This is due to the wavelength ( $\lambda=2 \pi / \omega=628$ for $\omega=0.01$ ) being too large compared to the spread of the observation region $(L=3)$, which is equivalent to having all the microphones at the same axial location, making it impossible to capture the wave form.

The study of the influence of both the number of randomly placed microphones $N$ and the observation spread $L$ is realized as follow. For each couple $(N, L)$, the inference process is performed at $\omega=1$ and $\omega=5$, other parameters being equal to the values taken in Sec. 4.1. To characterize the uncertainty on the identification, the standard deviation associated to the posterior densities of the parameters are calculated. The higher the standard deviation, the greater the uncertainty of a parameter. Our initial analysis showed that an increase in either $N$ or $L$ would, as expected, result in a lower standard deviation of the considered modes (results are similar at all frequencies). Moreover, it appeared that $N \times L$ was the most relevant parameter, sufficient to predict the order of magnitude of the standard deviation. To back-up this claim, the standard deviation of two modal amplitudes are shown in Fig. $7 \mathrm{~b}$ along with the associated best linear fits. For a practical antenna design, it is thus relevant to consider that the uncertainty (i.e., the standard deviation of the modal amplitudes) obeys a law of the type

$$
\log (\text { St. D. }) \propto-\alpha \cdot \log (N \times L),
$$

which was obtained by averaging the linear regression on the different modes. At $\omega=1, \alpha \approx-0.5$ while at $\omega=5$, $\alpha \approx-0.8$ for the $(0,0)$ mode and $\alpha \approx-0.7$ for the $(3,0)$ mode. Note that the lower standard deviation observed for the $(3,0)$ mode compared to that of the $(0,0)$ mode is explained in the same manner as in Sec. 4.1 .

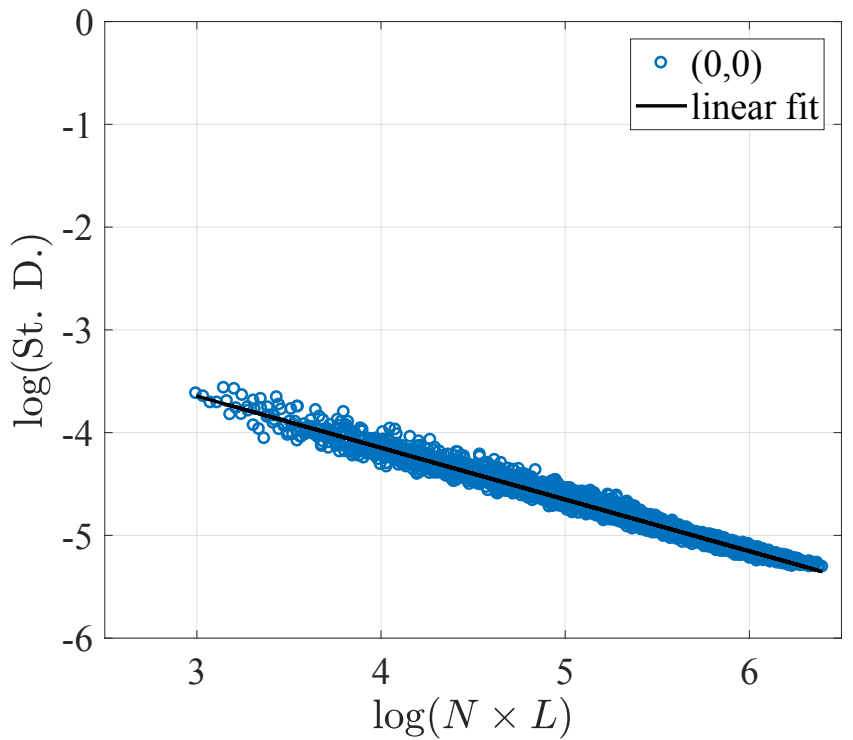

(a) $\omega=1$

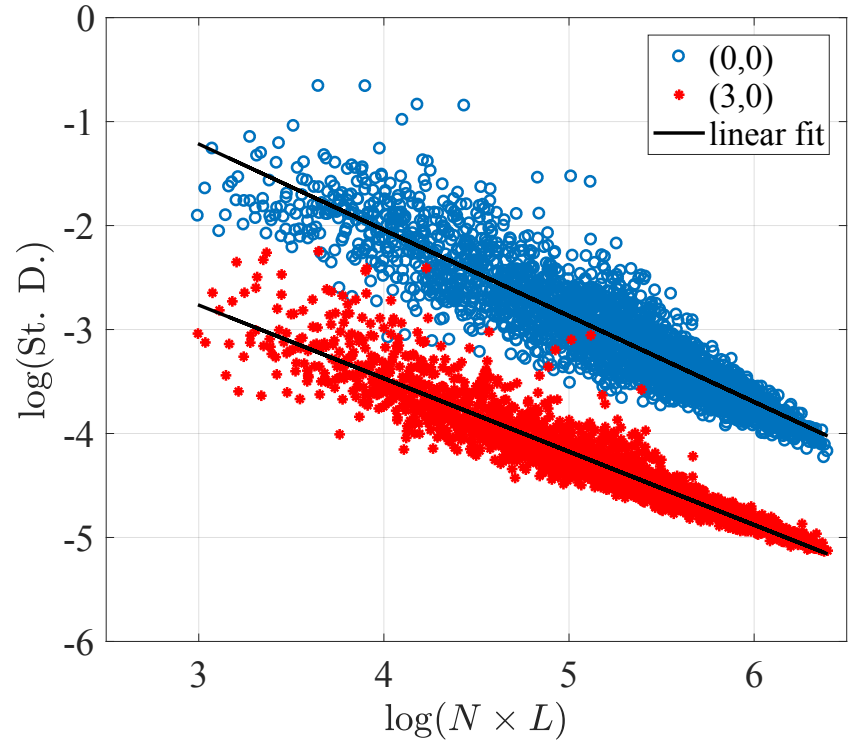

(b) $\omega=5$

Figure 7: Standard deviation of modal coefficient amplitudes as a function of the observation density, with randomly placed microphones 


\subsection{Case with a shear grazing unknown flow}

In the case where a grazing flow is present, the inference is quite similar to the one performed in the no flow case. The main difference is that the flow parameters $M_{b}$ and $\delta$ of Eq. 14 are now considered as unknown parameters to be inferred. At each iteration of the optimization process, the eigenvalue problem of Sec. 2.3 is recreated and solved, while it was created only once in the no flow case to compute the modal matrix $M$ (see Eq. 15). Due to the need for a refined mesh to capture the shear of the flow profile near the wall boundary, the procedure is more computationally demanding. A future prospect could be the use of surrogate models so as to predict the wavenumber values at lower cost.

In the present work, only two inferences are carried out for demonstrative purposes, at $\omega=1$ and $\omega=5$, with a given "true" flow profile having $M_{b}=0.2$ and $\delta=0.1$ (see dashed red curve in Fig. 2), and $N=100$ microphones spread randomly over a length $L=3$. Identification results are given in Table 1 (only two modes shown for conciseness in the case of $\omega=5$ ), where the true value of each parameter is compared to the inferred MAP and standard deviation. Since the flow parameters are now considered as random variables, the resulting flow profiles are shown in Fig. 8 for the case where $\omega=5$. Overall, the inference shows MAP values very close to the true value (within 2 standard deviations), for both the flow parameters and the modal coefficients, which validates the technique. The only parameter wrongly identified is the model standard deviation $\sigma$ when $\omega=5$.

While it is not its purpose, the inference method could interestingly be used to evaluate an experimental flow profile, given only an observation of the pressure field at the duct boundary. We note that these parameters are readily measurable by other, more classical, measuring techniques (hot wire probing). However, the present method gives an idea of the sensitivity of these parameters relatively to the modal decomposition.

At low frequencies, when only one mode is present $(\omega=1)$, the standard deviation of the identified flow parameters is quite large, too large actually to still warrant a Gaussian hypothesis. A Gaussian posterior density function for either $M_{b}$ or $\delta$ having these standard deviations would extend beyond the physical limits enforced by the prior $\left(M_{b}>0, \delta>0\right)$, thus invalidating the hypothesis. It is not surprising that when only one mode is present, the current method fails to accurately identify the flow parameters. $M_{b}$ and $\delta$ have a coupled effect of the first mode wavenumber, which results in their probability density functions being extremely correlated, hence the difficulty encountered during their identification.

\begin{tabular}{lccccc}
\hline & & \multicolumn{2}{c}{$\omega=1$} & \multicolumn{2}{c}{$\omega=5$} \\
Parameter & True value & MAP & St. D & MAP & St. D \\
\hline \hline$\sigma$ & 0.07 & 0.074 & $4.8 \cdot 10^{-3}$ & 0.14 & 0.01 \\
$M_{b}$ & 0.2 & 0.21 & 0.12 & 0.194 & $3.4 \cdot 10^{-3}$ \\
$\delta$ & 0.1 & 0.103 & 0.87 & 0.09 & $2.0 \cdot 10^{-2}$ \\
$\left|A_{0,0}\right|$ & 1 & 1.00 & $8.5 \cdot 10^{-3}$ & 1.01 & $2.0 \cdot 10^{-2}$ \\
$\angle_{0,0}^{+}$ & 0.79 & 0.77 & $2.0 \cdot 10^{-2}$ & 0.81 & $2.3 \cdot 10^{-2}$ \\
$\left|B_{0,0}\right|$ & 0.36 & 0.36 & $8.8 \cdot 10^{-3}$ & 0.35 & $1.6 \cdot 10^{-2}$ \\
$\angle_{0,0}^{-}$ & 0.20 & 0.18 & $5.0 \cdot 10^{-2}$ & 0.22 & $4.8 \cdot 10^{-2}$ \\
$\left|A_{3,0}\right|$ & 1 & - & - & 1.00 & $1.7 \cdot 10^{-2}$ \\
$\angle_{3,0}^{+}$ & 0.79 & - & - & 0.78 & $2.1 \cdot 10^{-2}$ \\
$\left|B_{3,0}\right|$ & 0.36 & - & - & 0.35 & $1.7 \cdot 10^{-2}$ \\
$\angle_{3,0}^{-}$ & 0.20 & - & - & 0.26 & $6.1 \cdot 10^{-2}$ \\
\hline
\end{tabular}

Table 1: Inference results for the shear grazing flow case

\section{Conclusion}

A statistical inference modal decomposition of in-duct pressure fields has been described. The technique can be regarded as a direct extension of the deterministic method of Åbom [11. The initial inversion problem is recast as a Bayesian inference problem, where parameters of interest (modal coefficients) are considered as random variables. 


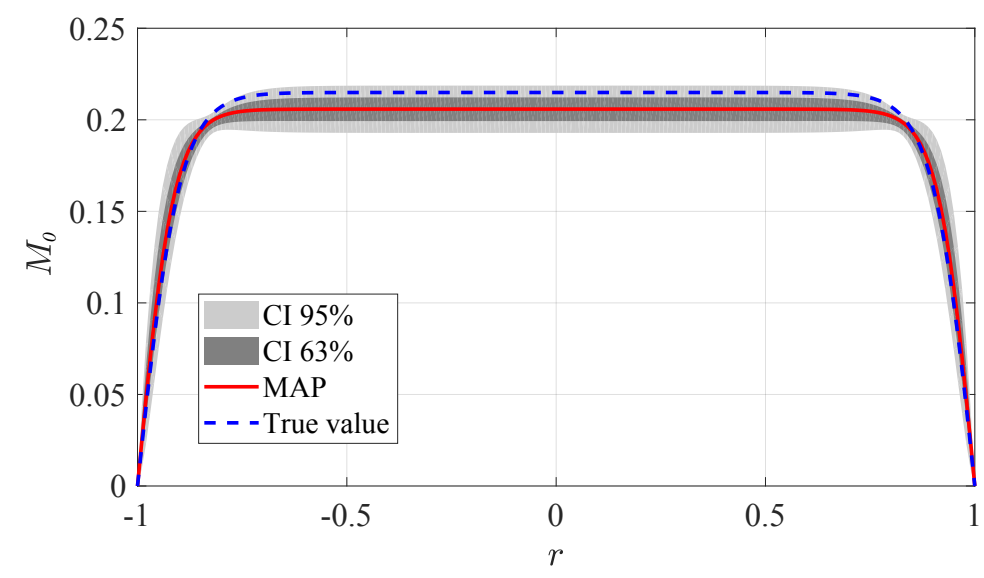

Figure 8: Comparison between inferred flow profile and true profile at $\omega=5$

A formulation based on Bayes theorem is advantageous since it allows the taking into account of both measurement and model uncertainties in a robust way, as well as possible prior knowledge on the unknown parameters.

An extension of the technique was introduced to take into account a general shear flow, while considering the flow profile to be uncertain. The technique was successfully applied to both the no-flow case (Sec. 4.1) and the case where a shear flow is present (Sec. 4.3), showing the possibility to infer the flow profile parameters and the modal coefficients simultaneously. The fact that the flow parameters could be identified so precisely at high frequencies $(\omega=5)$ means that they have a strong sensitivity relatively to the observation (pressure field at the wall boundary). When only one mode is present, the large uncertainty of the identified flow parameters suggest that plane-wave mode analyses should not be adversely affected by a uniform flow hypothesis. A future work shall study the influence of a uniform flow hypothesis on the modal identification in more details.

In the no flow case, a particular observation was made. Higher order modes seem "easier" to identify at high frequencies, compared to low order modes. This phenomenon, which might seem counter-intuitive to the experimentalist, was explained by an increased correlation between low order modes, caused by a localization of the wavenumbers of these modes at high frequency.

The inference results were also used in order to show the influence of the number of microphone locations $N$ and the spread of the measurement area $L$ on the uncertainty obtained on the modal coefficients amplitudes. It appeared that the design parameter of interest is the product $N \times L$, which displays an exponential relationship with the credibility intervals of the identified modal coefficients. Overall, the present approach allows practical design assessments relative to industrial needs for in-duct antenna design for acoustic modal decomposition.

\section{References}

[1] T. Schultz, L. N. Cattafesta III, M. Sheplak, Modal decomposition method for acoustic impedance testing in square ducts, The Journal of the Acoustical Society of America 120 (6) (2006) 3750-3758. doi:10.1121/1. 2360423 .

[2] J. L. Horner, Y. Hu, A hybrid modal decomposition approach for higher-order modes in circular ducts, Applied Acoustics 74 (1) (2013) 122-133. doi:10.1016/j.apacoust.2012.06.018.

[3] Y.-H. Heo, J.-G. Ih, H. Bodén, In-duct identification of fluid-borne source with high spatial resolution, Journal of Sound and Vibration 333 (23) (2014) 6074-6089. doi:10.1016/j.jsv.2014.07.005.

[4] T. Suzuki, B. J. Day, Comparative study on mode-identification algorithms using a phased-array system in a rectangular duct, Journal of Sound and Vibration 347 (2015) 27-45. doi:10.1016/j.jsv.2013.06.027

[5] A. Sanada, Extension of the frequency range of normal-incidence sound-absorption-coefficient measurement 
using four or eight microphones, Acoustical Science and Technology 38 (5) (2017) 261-263. doi:10.1250/ast. 38.261 .

[6] R. Boucheron, S. Amailland, J.-h. Thomas, C. Pezerat, D. Frechou, L. Briancon-Marjollet, Experimental modal decomposition of acoustic field in cavitation tunnel with square duct section, in: Proceedings of Meetings on Acoustics 173EAA, Vol. 30, ASA, 2017, p. 070002. doi:10.1121/2.0000570.

[7] P. Sijtsma, Feasibility of in-duct beamforming, in: 13th AIAA/CEAS Aeroacoustics Conference (28th AIAA Aeroacoustics Conference), 2007, p. 3696. doi:10.2514/6.2007-3696.

[8] U. Tapken, B. Pardowitz, M. Behn, Radial mode analysis of fan broadband noise, in: 23rd AIAA/CEAS Aeroacoustics Conference, 2017, p. 3715. doi:10.2514/6.2017-3715.

[9] L. Enghardt, A. Holewa, U. Tapken, Comparison of different analysis techniques to decompose a broad-band ducted sound field in its mode constituents, in: 13th AIAA/CEAS Aeroacoustics Conference (28th AIAA Aeroacoustics Conference), 2007, p. 3520. doi:10.2514/6.2007-3520.

[10] S. Fauqueux, R. Davy, Modal deconvolution method in a finite circular duct, using flush-mounted microphones, in: 2018 AIAA/CEAS Aeroacoustics Conference, 2018, p. 3927. doi:10.2514/6.2018-3927

[11] M. Åbom, Modal decomposition in ducts based on transfer function measurements between microphone pairs, Journal of Sound and Vibration 135 (1) (1989) 95-114. doi:10.1016/0022-460X (89)90757-8.

[12] J. Chung, D. Blaser, Transfer function method of measuring in-duct acoustic properties. ii. experiment, The Journal of the Acoustical Society of America 68 (3) (1980) 914-921. doi:10.1121/1.384779.

[13] T. Schultz, M. Sheplak, L. N. Cattafesta III, Uncertainty analysis of the two-microphone method, J. Sound Vib. 304 (1-2) (2007) 91-109. doi:10.1016/j.jsv.2007.02.015.

[14] J. Auger, J. Ville, Flow effects on measurement of the modal decomposition of acoustic field in a hard wall cylindrical duct, in: Aero-and Hydro-Acoustics, Springer, 1986, pp. 437-443. doi:10.1007/978-3-642-82758-7_ 41.

[15] C. Morfey, Sound transmission and generation in ducts with flow, Journal of Sound and Vibration 14 (1) (1971) 37-55. doi:10.1016/0022-460X(71)90506-2.

[16] S. Rienstra, A. Hirschberg, An introduction to acoustics, Eindhoven University of Technology (2013) 151-154.

[17] G. Boyer, E. Piot, J.-P. Brazier, Theoretical investigation of hydrodynamic surface mode in a lined duct with sheared flow and comparison with experiment, Journal of Sound and Vibration 330 (8) (2011) 1793-1809. doi:10.1016/j.jsv.2010.10.035

[18] S. Rienstra, G. Vilenski, Spatial instability of boundary layer along impedance wall, in: 14th AIAA/CEAS Aeroacoustics Conference (29th AIAA Aeroacoustics Conference), 2008, p. 2932. doi:10.2514/6.2008-2932.

[19] D. Khamis, E. J. Brambley, Viscous effects on the acoustics and stability of a shear layer over an impedance wall, Journal of Fluid Mechanics 810 (2017) 489-534. doi:10.1017/jfm.2016.737.

[20] J. Y. Chung, D. A. Blaser, Transfer function method of measuring in-duct acoustic properties. I. Theory, J. Acoust. Soc. Am. 68 (3) (1980) 907-913. doi:10.1121/1.384778.

[21] R. C. Smith, Uncertainty quantification: theory, implementation, and applications, Vol. 12, Siam, Philadelphia, 2013.

[22] E. Laloy, J. A. Vrugt, High-dimensional posterior exploration of hydrologic models using multiple-try dream(zs) and high-performance computing, Water Resour. Res. 48 (1) (2012) n/a-n/a, w01526. doi: 10.1029/2011WR010608.

[23] M. Lavieille, S. Le-Saint, Impedance eduction of liners in no-flow condition and based on multimodal excitation, in: 23rd AIAA/CEAS Aeroacoustics Conference, 2017, p. 3180. doi:10.2514/6.2017-3180 